\section{Endoscopic Diagnosis of Gastroduodenal Intussusception}

Gastroduodenal intussusception is a rare condition caused by the prolapse of a gastric tumor and subsequent invagination of the full thickness of a portion of the gastric wall into the duodenum $(1,2)$. Nearly all diagnoses have been made by radiography, or after surgery $(3,4)$. In spite of the widespread use of endoscopy in the diagnosis of gastric lesions, the endoscopic features of gastroduodenal intussusception have rarely been reported. We present here the case of a patient with gastroduodenal intussusception of a lateral type, caused by a stromal-cell sarcoma, with emphasis on the endoscopic features.

A 65-year-old man was admitted, the chief complaint being hematemesis and tarry stool for one day. A physical examination and routine laboratory tests only revealed anemia. Upper gastrointestinal endoscopy disclosed abnormally convergent gastric folds, extending from the great curvature side and the anterior wall of the gastric body toward the antrum (Figure 1). The folds spread downward to the angle, and converged as a band-like structure at the distal antrum (Figure 2 a), terminating at a mass that partially occluded the pylorus (Figure $\mathbf{2 b}$ ). The surface of the tumor was smooth, but congested. The endoscopic diagnosis was a gastric tumor with gastroduodenal intussusception of a lateral type, according to the classification defined by Hobbs and Cohen (5). A barium meal study showed a distended stomach, with delayed emptying. Computed tomography revealed an intraluminal softtissue density from the bulb to the second portion of duodenum. Laparotomy revealed an incarcerated tumor at the pyloric ring, extending to the second portion of the duodenum. A dimpling on the serosa of the gastric body was also found. After reduction and gastrostomy, a tumor measuring $5 \times 5 \times 4 \mathrm{~cm}$ in size, with an ulceration at the tip, was found at the leading point of the intussusceptum. The tumor was resected, and pathological diagnosis showed a stromal-cell sarcoma.

The patient recovered smoothly after surgery. No tumor recurrence was found during a follow-up period of one year.

\section{References}

1. Rocobono XJ, Haskins RM. Gastroduodenal intussusception: report of two cases. Gastroenterology 1960; 38: 995-1002.

2. Lin F, Setya V, Signor W. Gastroduodenal intussusception secondary to a gastric lipoma: a case report and review of the literature. Am Surg 1992; 58: $772-4$.

3. Meyers MA. Gastroduodenal intussusception. Am J Med Sci 1967: 254: 347-55

4. Kleinhaus U, Weich YL, Maoz S. Gastroduodenal intussusception secondary to prolapsing gastric tumors. Gastrointest Radiol $1986 ; 11: 229-32$.

5. Hobbs WH, Cohen SE. Gastroduodenal intussusception due to a submucosal lipoma of the stomach. Am J Surg 1946; 71: 505 -18 .

\footnotetext{
T.-H. Lee', C.-H. Chen' ', J.-M. Wong', S.-C. Yu', C.-T. Shun ${ }^{3}$, T.-H. Wang', J.-T. Lin'

Dept. of Internal Medicine

${ }^{2}$ Dept. of Surgery

${ }^{3}$ Dept. of Pathology, College of Medicine, National Taiwan

University, Taipei, Taiwan
}

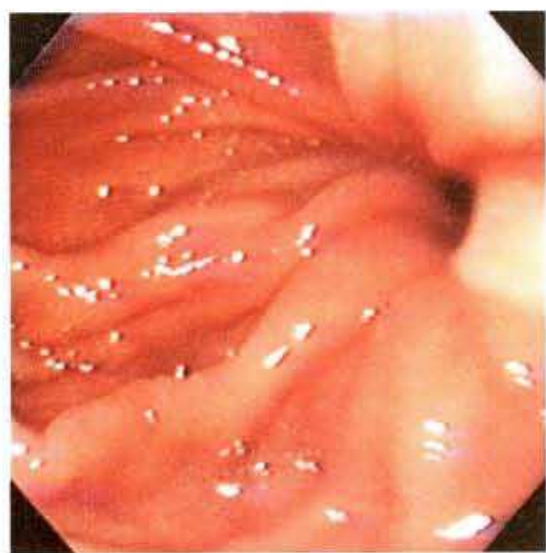

Figure 1: The endoscopic examination shows abnormally convergent gastric folds extending from the anterior wall and great curvature side of the gastric body toward the antrum.

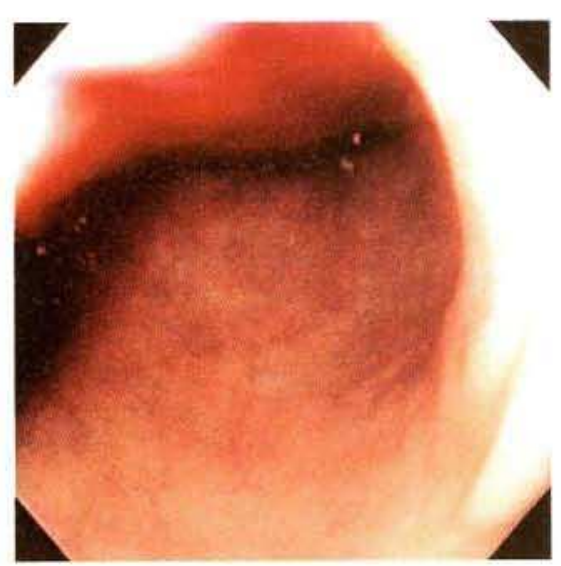

Figure 2a: The folds spread downward to the gastric angle, and converge as a band-like structure at the distal antrum.

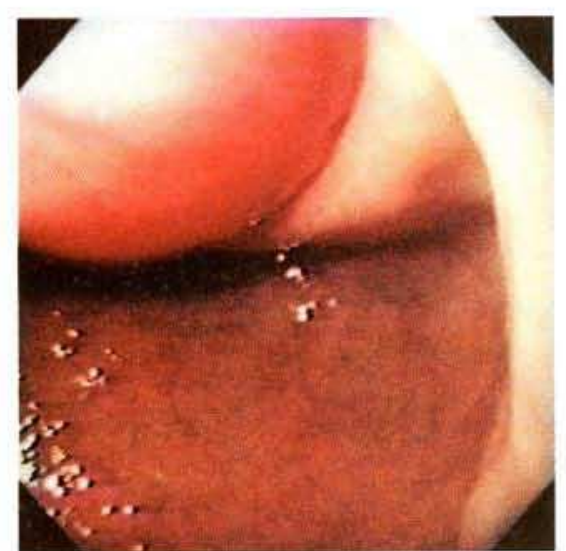

Figure $\mathbf{2} \mathrm{b}$ : Conver gent folds terminating at a mass, which is incarcerated in the pyloric ring
Corresponding Author

J.-T. Lin, M.D.

Dept. of Internal Medicine

National Taiwan University Hospital

7 Chung-Shan South Road

Taipei

Taiwan 10016

Fax: $+886-2-3710791$ 